\title{
Laparoscopic management of primary abdominal pregnancy located on the sigmoid colon: A case report
}

\author{
A Benhaddou ${ }^{1, *}$, C Kouas $^{2}$ and S Mehaut ${ }^{3}$ \\ 1 Service de gynécologie et obstétrique, centre hospitalier de Vitry-le-François, 51300 Vitry-le-François, France \\ 2 Service d'anesthésie-réanimation, centre hospitalier de Vitry-le-François, 51300 Vitry-le-François, France \\ ${ }^{3}$ Service d'anatomopathologie, centre hospitalier de Troyes, 10000 Troyes, France
}

\begin{abstract}
Abdominal pregnancy (AP) is rare with an estimated incidence of $1 / 10000$ births and only $1.4 \%$ of ectopic pregnancies. Primary abdominal pregnancy (PAP) is the least common form of the AP. We present a case of PAP in a 22 year-old woman who was admitted in our department with diffuse abdominal pain, predominant in the pelvic area with a good general condition. The serum BhCG level was $1643 \mathrm{mIU} / \mathrm{mL}$. Ultrasonographic exploration showed an empty uterus with a free fluid and hyperechoic mass in the Douglas pouch. An emergency laparoscopic surgery showed abdominal pregnancy located on the sigmoid colon. Resection of the PAP and hemostasis were performed by laparoscopy. To our knowledge, only four cases of PAP implanted on the sigmoid colon have been reported to date but only two have been treated by laparoscopy. AP must be diagnosed and treated early to avoid complications such as hemorrhage due to placental implantation that is a potentially life-threatening condition.
\end{abstract}

Keywords: primary abdominal pregnancy; laparoscopy; ultrasonography; RMI; embolization; methotrexate

\section{Introduction}

Abdominal pregnancy (AP) has been defined as a pregnancy occurring within the peritoneal surface outside the uterus, fallopian tubes and ovaries. This is a rare entity that represents only $1.4 \%$ of all ectopic pregnancies. There are two forms of AP: secondary and primary AP. The primary abdominal pregnancy (PAP) is the least common form of AP. To our knowledge, only four PAP implanted on the sigmoid colon have been reported in the world literature to date.

This report concerns a rare case of PAP, arising on the sigmoid fringe and treated by laparoscopy.

\section{Case report}

A twenty-two-year-old woman, gravida 2, para 0, was admitted to our emergency room for diffuse abdominal pain predominant in the pelvic area and lasting for five days. The patient had minimal bleeding prior to 15 days, but the menstruation had begun four weeks before this episode. The woman does not use any contraceptive method. In her medical history, there was a miscarriage and an abortion, both treated by the medical method in 2010 . She underwent a laparoscopy in 2011 for a hemorrhagic cyst of the right ovary.

On admission on March 30, 2014, there was a moderate anemia, a stable hemodynamic status and a clinical examination noted a diffuse abdominal tenderness predominant in the pelvic area. Ultrasonographic examination showed an empty uterus with fluid and echogenic mass in Douglas pouch (Figure 1). The BhCG serum level was $1643 \mathrm{mlU} / \mathrm{mL}$, a hemoglobin level of $55 \mathrm{mmol} / \mathrm{L}$ and platelet count at $110.103 / \mu \mathrm{L}$. The clinic findings suggested an ectopic pregnancy, and then an emergency laparoscopy was performed. Exploration of the pelvis revealed a hemoperitoneum of approximately $600 \mathrm{ml}$ of blood and blood clots which was aspirated. The uterus, both fallopian tubes and ovaries appeared normal (Figure 2a) but an embryo attached to the sigmoid arises from the Douglas pouch (Figure 2b). A removal of the embryo and hemostasis were done by Ultracision.

The postoperative course was uneventful and the patient was discharged the day after surgery. The ßhCG level had returned to normal within two weeks after surgery.

*Corresponding author: Dr. Abdelhaq Benhaddou, Department of Gynecology and Obstetrics, Vitry-le-François Hospital Center, 51300 Vitryle-François, France. E-mail: a.benhaddou@ch-vitrylefrancois.fr

Received 16 September 2016 Revised 12 November 2016 Accepted 24 November 2016 Published 29 November 2016

Citation: Benhaddou A, Kouas C, Mehaut S. Laparoscopic management of primary abdominal pregnancy located on the sigmoid colon: A case report. J Mod Hum Pathol. 2016; 1(8):67-69. DOI: 10.14312/2397-6845.2016-10

Copyright: (c) 2016 Benhaddou A, et al. Published by NobleResearch Publishers. This is an open-access article distributed under the terms of the Creative Commons Attribution License, which permits unrestricted use, distribution and reproduction in any medium, provided the original author and source are credited. 


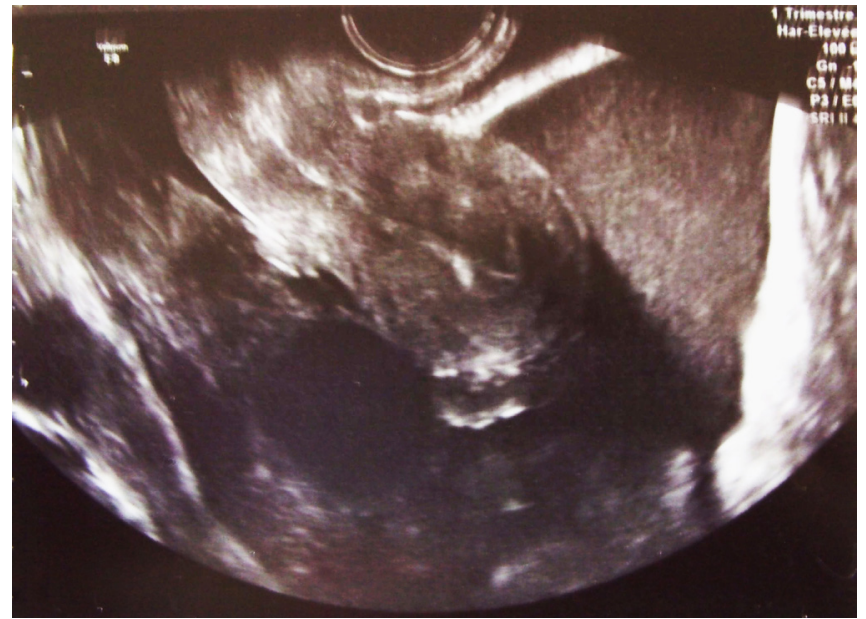

Figure 1 Ultrasonographic imaging of the primary abdominal pregnancy: Uterus is empty and bleeding with echoic image in the Douglas pouch.

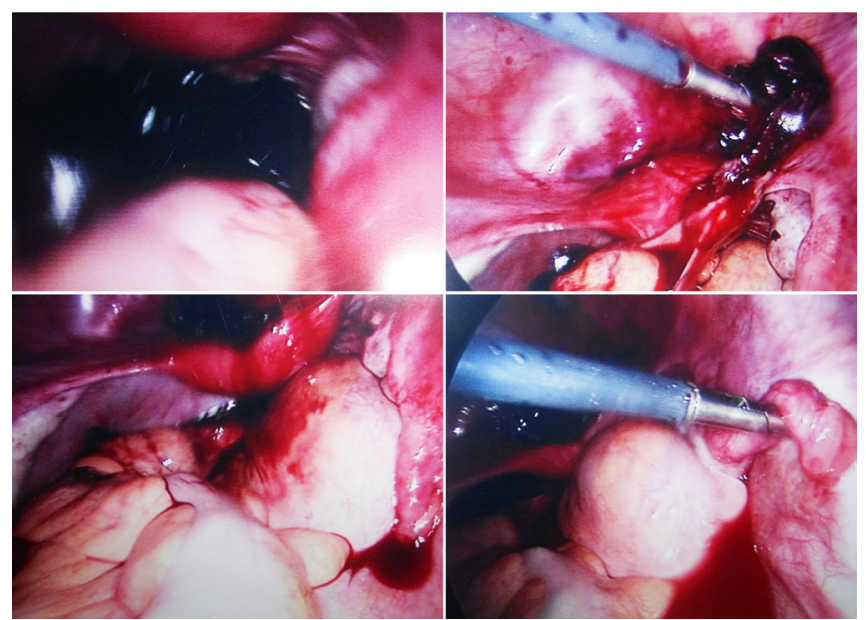

Figure 2a Abdominal pregnancy: note the fallopian and ovaries integrity.

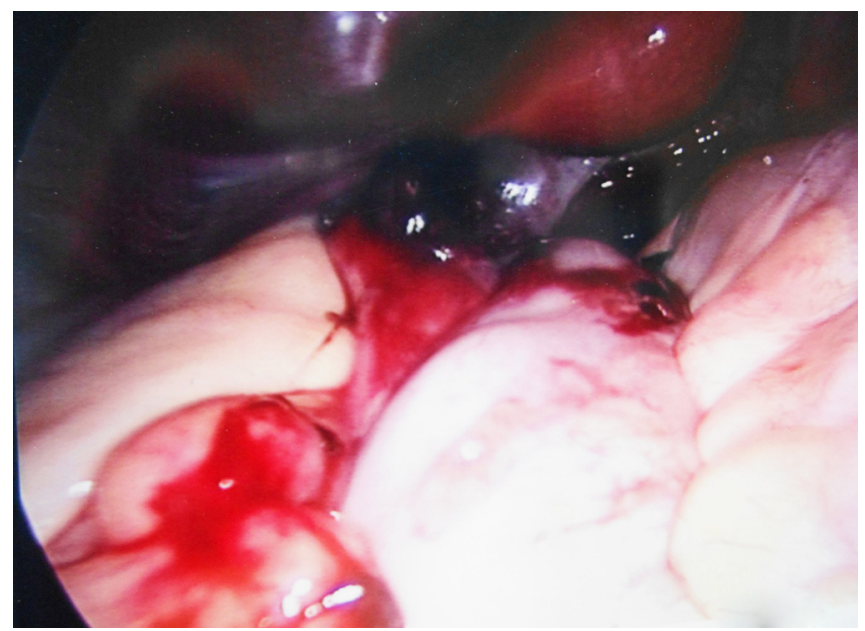

Figure $\mathbf{2 b}$ Abdominal pregnancy: note the implantation of the embryo on the sigmoid colon.

Histologic examination of the tissue confirms the diagnosis of abdominal pregnancy implanted on the sigmoid fringe with chorionic villi (Figure 3).

\section{Discussion}

Abdominal pregnancy (AP) is a rare entity. Its incidence is estimated at one per 10,000 births and represents $1.4 \%$ of all ectopic pregnancies (EP). Its incidence is probably higher

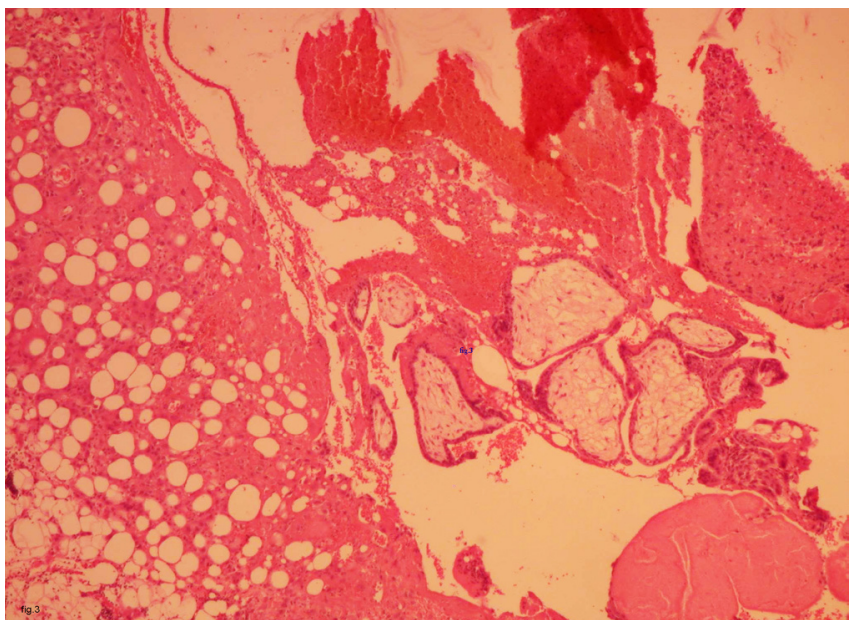

Figure 3 Histological image: implantation site on adipose tissue of sigmoid fringe with chorionic villi.

in developing countries because of the high frequency of pelvic infections and a low socioeconomic level [1, 2]. In their study, Bang Ntamack, et al. report an incidence of $1 / 4447$ births [1]. Infertility and IUD contraception are also found among the risk factors of the AP [1-5].

There are two forms of AP: primary and secondary AP. The latter is usually due to tubal abortion or rupture of a tubal pregnancy implanting secondarily in the peritoneal cavity. It represents the majority of AP. AP secondary to uterine perforation is exceptional. The primary abdominal pregnancy (PAP) meets the Studdiford criteria [6]: «no uteroperitoneal fistula, absence of tubal and ovarian lesions and pregnancy related exclusively to the peritoneal surface and early enough to eliminate a secondary implantation after a first tubal implantation». Because the management of both situations is the same, another classification based on gestational age has been proposed. This classification separates early AP of less than 20 weeks from advanced AP evolving beyond 20 weeks of gestational age.

The PAP is a very rare entity that includes our case. Approximately twenty cases of PAP have been reported in the world literature, mostly located on the omentum, but only four cases of sigmoid implantation [3, 7, 8 ] of which only two were treated by laparoscopy as in our case [3]. The majority of AP is located in the pelvic area, but some cases have reported AP on the liver and spleen [9, 10,4].

Maternal mortality associated with AP is 7.7 times greater than those associated with other ectopic pregnancies and 90 times higher than those associated with intrauterine pregnancies [5, 11, 12]. Poole et al. [11], postulate that the high risk of mortality in AP is due to delay in diagnosis.

Hallatt and Grove [13], recommend immediate surgery as soon as the diagnosis of AP is established. According to these authors: "A risk of intra-abdominal catastrophe does not allow for a period of watchful waiting, nor does the high fetal mortality and malformation rate warrant delay for fetal indication."

AP develops poor clinical features that make it difficult to diagnose. There are several ways to diagnose AP, but many 
are too invasive or insufficient for an appropriate diagnosis. Ultrasonography is the only non-invasive examination to diagnose an ectopic pregnancy. Regardless of the method used, the diagnosis of AP remains difficult.

When laparoscopy is performed for suspected ectopic pregnancy and the tubes are normal, an exploration of the whole peritoneal surface must be meticulous to rule out an AP. In fact, at least 5 cases of AP that were misdiagnosed at the first laparoscopy were reported in the world literature $[4,5,10,14,15]$. However, two cases were formidable pitfalls $[14,15]$. In the first case, the emergency laparoscopy discovered an ovarian bleeding evoking an ovarian pregnancy, while the AP was located on the ascending colon. The diagnosis was done by MRI [14]. The second case was a transfer of three embryos. Laparoscopy discovered a tubal pregnancy but two days after the first laparoscopy, a second procedure was performed because of an increasing ßhCG level, and then showed an abdominal pregnancy located on spleen [15]. Other studies have highlighted the difficulty in diagnosing AP because of their rarity and uncommon clinical findings. Moonen-Delarue et al. [11], recommend to "think ectopic" because of an unfortunate experience of two successive misdiagnosed APs. In their study, Atrash et al. [16] analyzed the American Registry of maternal deaths related to AP and found that only 2 of the 11 patients who died from AP were pregnant of less than 12 weeks CG level gestation. They also noted that of all 126 previously reported abdominal pregnancy related deaths with known gestational age, 13\% presented before 12 weeks.

AP is a rare entity that necessitates early diagnosis in order to avoid complications, such as hemorrhage, that can be dramatic for both the mother and the fetus [12, 16]. The prognosis of this disease depends exclusively on the placental implantation, the removal of which is often difficult or impossible due to the implantation over major vessels, bowel, or other vital structures [15-18, 9]. The placenta is often left in situ.

MRI, when available, shows the exact location of the placenta and its implantation site to assess the possibility of its removal. If this is not feasible, selective embolization of vessels supplying the placenta and / or in situ administration of methotrexate may be discussed. However, some unsuccessful diagnoses of placental implantation sites by MRI have been reported [9].

When removal of the placenta is necessary, bowel preparation, assurance of sufficient blood products, and availability of a multidisciplined surgical team is required. Should such resources not be available, elective transfer of a woman with a known advanced extrauterine pregnancy to a level III hospital is appropriate to prevent a cataclysmic hemorrhage.

\section{Conclusion}

Ultrasonography, a serum ßhCG level and laparoscopic surgery have really improved management and prognosis of AP. Thus, over the last 20 years, the mortality rate associated with AP has decreased from $20 \%$ to $5 \%$. Laparoscopy remains the treatment of choice in early
AP. It allows for better recovery and fewer postoperative complications than laparotomy. The patient had successful pregnancy one year after the operation and gave birth to healthy baby on 2016. In advanced abdominal pregnancy (AAP), only laparotomy allows for adequate treatment. A placental implantation is the cornerstone of the AP which can be life-threatening because of the hemorrhage associated with its removal or infection if left in situ. The ideal is to remove the placenta to avoid serious complications. The bleeding risk can be reduced by selective embolization and / or using methotrexate in situ preoperatively. If the placenta is left in situ, the patient must continue to be monitored for possible complications such as infections or secondary peritonitis due to placental necrosis.

\section{Conflicts of interest}

The authors declare no conflicts of interest.

\section{References}

[1] Bang Ntamack JA, Ngou JP, Mve Ngou C, Sima Ole B, Sima Zue A, et al. Abdominal pregnancy in Libreville from 1999 to 2009. J Gynecol Obstet Biol Reprod. 2012; 41(1):83-87.

[2] Aliyu LD, Ashimi AO. A multicentre study of advanced abdominal pregnancy: A review of six cases in low resource settings. Eur J Obstet Gynecol Reprod Biol. 2013; 170(1):33-38.

[3] Uzan M, Von Theobald P, Lucas V, Barjot P, Liegeois P, et al. Ectopic abdominal pregnancy: Two cases treated by laparoscopic surgery. J Gynecol Obstet Biol Reprod (Paris). 1993; 22(4):429-430.

[4] Martellie F, De Carolis C, Parisi C, Piccione E. Neglected primary omental pregnancy after laparoscopic and medical treatment: A difficult diagnosis? Case Rep Obstet Gnecol. 2013; 2013:207-307.

[5] Koo HS, Bae JY, Kang IS, Koong MK, Kim HO, et al. Laparoscopic management of early primary peritoneal pregnancy: A case report. Clin Exp Reprod Med. 2011; 38(2):109-114.

[6] Studdiford WE. Primary peritoneal pregnancy. Am J Obstet Gynecol. 1942; 44(3):487-491.

[7] Ganeshselvi P, Cherian D, Champ S, Myerson N. Primary abdominal pregnancy implanted on the sigmoid colon. J Obstet Gynaecol. 2003; 23(6):667.

[8] Makinen J. Histologically verified primary peritoneal pregnancy with implantation in the sigmoid mesenterium. Eur J Obstet Gynecol Reprod Biol. 1986; 22(3):171-174.

[9] Poole A, Haas D, Magann EF. Early abdominal ectopic pregnancies: A systematic review of the literature. Gynecol Obstet Invest. 2012; 74(4):249-260.

[10] Cristalli B, Guichaoua H, Heid M, Izard V, Levardon M. Abdominal ectopic pregnancy. Limits of laparoscopic treatment. J Gynécol Obstét Biol Reprod. 1992; 21(7):751-753.

[11] Moonen-Dlarue MW, Haest JW. Ectopic pregnancy three times in line of which two advanced abdominal pregnancies. Eur J Obstet Gynecol Reprod Biol. 1996; 66(1):87-88.

[12] Laher AE, Goldstein LN, Wells MD, Mahomed Z, Gihwala R, et al. Perimortem laparotomy in a patient with a ruptured intra-abdominal pregnancy. Afr J Emerg Med. 2013; 3(4):178-181.

[13] Halatt JG, Grove JA. Abdominal pregnancy: A study of twenty one consumptive cases. Am J Gynecol Obstet. 1985; 152(4):444-449.

[14] Yi KW, Yeo MK, Shin JH, Kim KA, Oh MJ, et al. laparoscopic management of early omental pregnancy detected by magnetic resonance imaging. J Minim Invasive Gynecol. 2008; 15(2):231-234.

[15] Kitade M, Takeuchi H, Kikuchi I, Shimanuki H, Kumakiri J, et al. A case of simultaneous tubal-splenic pregnancy after assisted reproductive technology. Fertil Steril. 2005; 83(4):1042.

[16] Atrash HK, Friede A, Hogue CJ. Abdominal pregnancy in the United States: Frequency and maternal mortality. Obstet Gynecol. 1987; 69(3 Pt 1):333-337

[17] Ma J, Zhou C, Duan Z, Jiang Y. Successful management of primary hepatic pregnancy with selective hepatic artery embolization and intraarteial methotrexate infusion. Int J Gynaecol Obstet. 2013; 122(1):7879.

[18] Gang G, Yudong Y, Zhang G. Successful laparoscopic management of early splenic pregnancy: Case report and review of literature. J Minim Invasive Gynecol. 2010; 17(6):794-797. 